\title{
Charmonium and D mesons in hadronic matter
}

\author{
G. Krein \\ Instituto de Física Teórica, Universidade Estadual Paulista \\ Rua Dr. Bento Teobaldo Ferraz, 271 - Bloco II, 01140-070 São Paulo, SP - Brazil
}

\begin{abstract}
We discuss two aspects of charmonium in medium. First, we present results of a recent study that compares the phenomenology of charmonium spectroscopy using smooth and sudden string breaking potentials. Next, we present results of a study that explores the possibility that $J / \Psi$ might be bound in a large nucleus through the excitation of a color singlet intermediate states of $D$ and $D^{*}$ mesons with density masses.
\end{abstract}

Keywords: Charm in matter, string breaking, chiral symmetry restoration

PACS: $21.85 .+\mathrm{d}, 21.65 \mathrm{Jk}, 21.65 . \mathrm{Qr}, 24.85 .+\mathrm{p}$

\section{INTRODUCTION}

The study of temperature $(T)$ and baryon density $(\rho)$ dependence of properties of charmed mesons in hadronic matter is of interest in different contexts. One example is the conjectured suppression of $J / \Psi$ production in relativistic heavy ions collisions, where screening of the long range confining potential due to deconfined light quarks of the hot and dense medium would make impossible the formation of the charmonium state. However, a $J / \Psi$ suppression can also occur via dissociation due to collisions of these mesons with hadrons in the medium and therefore the initial hope [1] to use $J / \Psi$ suppression as a diagnosis tool for the formation of a quark-gluon plasma is undermined. Another focus of interest is the possibility of the existence of an exotic nuclear bound state in which a charmonium state $(c \bar{c})$ produced close to threshold is captured in matter [2]. Such a bound state would reveal the importance of multiple-gluon mediated processes, like color van der Waals forces, since charmonium and ordinary hadrons (formed by $u$ and $d$ quarks) have no quarks in common. Still another area of interest refers to studies of chiral symmetry restoration at finite $T$ and $\rho$ in D mesons, which are composed of one light quark ( $u$ or $d$ ) and one heavy $c$ quark. The chiral properties of $u$ and $d$ quarks are much more sensitive to $T$ and $\rho$ than $c$ quarks and there is hope one can learn about chiral restoration studying the interactions of $\mathrm{D}$ mesons in matter.

In the present communication we will discuss two aspects of charmonium in medium. In the next Section we use a potential model to present results of a study [3] that compares the phenomenology of charmonium spectroscopy at finite temperature using smooth and sudden string breaking potentials. In the following Section, we present results of an ongoing study [4] of the possibility that $J / \Psi$ is bound in a large nucleus through the excitation of a color singlet intermediate states of $D$ and $D^{*}$ mesons with density dependent masses.

CP1265, VIII Latin American Symposium on Nuclear Physics and Applications edited by R. Alcaron, H. F. Arellano, P. L. Cole, and A. J. Kreiner

(C) 2010 American Institute of Physics 978-0-7354-0814-2/10/\$30.00 


\section{SMOOTH VERSUS SUDDEN STRING BREAKING}

As mentioned above, color screening of the interquark potential by deconfined light quarks in a hot and dense medium was thought to prevent the formation of $c \bar{c}$ bound states. In vacuum, color screening occurs as the interquark distance in a $c \bar{c}$ bound state is increased and leads to the breaking of the color string when the string is stretched beyond a certain limit. Such a string breaking phenomenon has been confirmed in lattice QCD simulations [5]. The simulations have shown in addition that the breaking of the string is quite sudden. For a bound $c \bar{c}$ pair in a medium, one would expect that such a sudden breaking would be even more dramatic, because of the existence of additional unconfined quarks in the medium. However, recent lattice results for $c \bar{c}$ correlation functions at finite temperature have shown $[6,7,8]$, somewhat surprisingly, that for temperatures $T$ well above the deconfinement temperature $T_{c}$ there remains strong correlations between the $c \bar{c}$ pairs. Such remaining correlations might indicate that $c \bar{c}$ bound states survive above the deconfinement temperature. The results motivated many studies of $c \bar{c}$ bound states in the context of potential models using some prescribed temperature dependence for the confining potential - for a recent review and a list of references, see Ref. [9]. In these studies, as a rule, the prescribed temperature dependence for the potential is motivated from lattice results for the finite temperature free energy. A problem with such an approach is that free-energy is not potential energy, free-energy contains an entropy contribution. Entropy much probably smooths out any sudden breaking of the string and as a consequence, calculations with such temperature-dependent potentials will predict a smooth temperature dependence for quarkonia properties close to the critical temperature .

Here we present results of a recent study [3] of $c \bar{c}$ bound state properties as calculated with smooth and sudden string breaking potentials - Ref. [3] calculated also $b \bar{b}$ properties. Qualitatively different results are obtained when using smooth or sudden stringbreaking potentials. Specifically, for a smooth string-breaking potential the following expression is used

$$
V_{\text {smooth }}(r)=\frac{\sigma}{\mu}\left(1-e^{-\mu r}\right)+V_{O G E}(r),
$$

with

$$
V_{O G E}(r)=-\frac{\alpha}{r}+\frac{\hbar^{2}}{m_{q} m_{\bar{q}}} \frac{\alpha}{r} \frac{e^{-r^{2} / r_{0}^{2}}}{r_{0}^{2}}\left(\vec{\sigma}_{1} \cdot \vec{\sigma}_{2}\right),
$$

where the phenomenological parameters $\sigma, \alpha$ and $r_{0}$ are fixed by fitting the quarkonium spectrum. Sudden string-breaking is implemented as

$$
V_{\text {sudden }}(r)= \begin{cases}\sigma r+V_{O G E}(r), & r<r_{b} \\ \sigma r_{b}+V_{O G E}\left(r_{b}\right), & r \geq r_{b},\end{cases}
$$

where $r_{b}$ is the inter-quark distance for which the string breaks. The values of the parameters of the potentials, shown in Table 1, were adjusted to obtain a reasonable description of the lowest-lying states. Given the parameters, the Schrödinger equation is solved numerically to obtain wave functions and masses. No specific model for the temperature 
dependence of the potentials is used, temperature dependence is mimicked by varying the screening parameters $\mu$ and $1 / r_{b}$, while all other parameters are maintained fixed.

TABLE 1. Parameters of the potentials in Eqs. (1)-(3).

\begin{tabular}{lrr}
\hline & smooth & sudden \\
\hline$\sigma\left(\mathrm{MeV} \mathrm{fm}^{-1}\right)$ & 1470 & 800 \\
$\alpha(\mathrm{MeV} \mathrm{fm})$ & 96 & 106 \\
$r_{0}(\mathrm{fm})$ & 0.38 & 0.36 \\
$\mu\left(\mathrm{fm}^{-1}\right)$ & 0.71 & - \\
$1 / r_{b}\left(\mathrm{fm}^{-1}\right)$ & - & 0.44 \\
$r_{0}(\mathrm{fm})$ & 0.38 & 0.36 \\
$m_{c}(\mathrm{MeV})$ & 1264 & 1385 \\
\hline
\end{tabular}

A large set of results for masses, radii and wave functions at the origin for ground states and excited states are presented in Ref. [3], for both charmonium $(c \bar{c})$ and bottomonium $(b \bar{b})$. Here we show results only for the r.m.s. radii for the charmonium states $\eta_{c}$ (solid), $J / \Psi$ (dashed) and $\chi_{c J}$. They are shown in Fig. 1, as a function of the screening parameters $\mu$ and $1 / r_{b}$. Clearly seen in this figure is the striking difference between the two situations. While for the smooth string-breaking potential the radii change smoothly and continuously, for the sudden string-breaking breaking they remain almost constant as the screening radius changes (temperature changes), but at a certain value of the screening radius (the critical temperature), the radii increase abruptly. It is interesting to mention that a similar behavior has been obtained for heavy-light mesons using a thermal Hilbert moment QCD sum rules [10, 11], a completely different approach from the one used here.
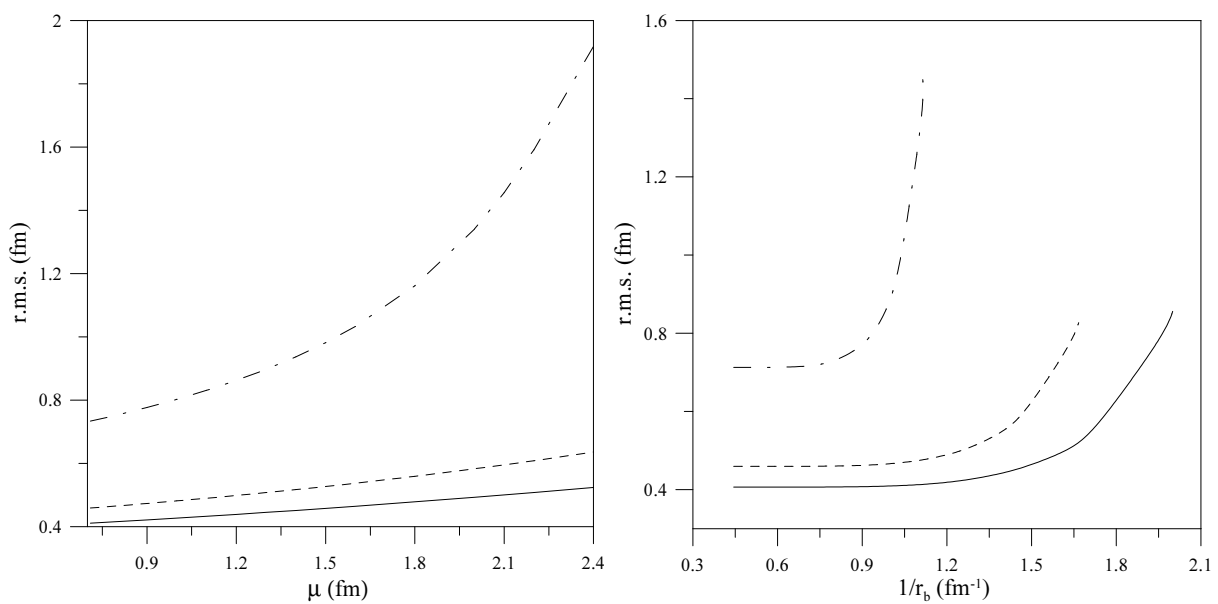

FIGURE 1. Screening mass dependence of the r.m.s. radii of $\eta_{c}$ (solid), $J / \Psi$ (dashed) and $\chi_{c J}$ (dasheddotted) $c \bar{c}$ bound states [3]. Left (right) panel is for the smooth (sudden) string-breaking potential. 
The dramatic, fast increase of the size of a bound state resembles a critical phenomenon and has impact in the phenomenology of quarkonia interactions in medium, in particular for scattering dissociation processes $[12,13,14,15]$ which proceed via constituent interchange. For short-range interactions, dissociation cross sections depend essentially on the degree of overlap of the wave functions of the scattering hadrons. Since the wave functions are normalized, an increase in the r.m.s radius has the effect of flattening out the wave functions and so the overlap of the wave functions of the colliding hadrons becomes less important and the probability of constituent interchange decreases. Such effects might have significant impact in the phenomenology of charmonium dissociation in medium and certainly deserves further study.

\section{$J / \Psi$ BINDING TO NUCLEAR MATTER}

In generic terms, the multiple-gluon mediated interactions of charmonium with ordinary matter can proceed via two mechanisms. One is via the excitation of a color-octet intermediate $c \bar{c}$ state [16]. The change in the mass of a charmonium state due to such an excitation can be calculated in a similar way the second order Stark effect in atomic physics is calculated. One of the most recent calculations of this kind was carried out in Ref. [17], with the result of $8 \mathrm{MeV}$ for the binding energy of $J / \Psi$ in nuclear matter. The other mechanism is via the excitation of an intermediate state where the $c$ and $\bar{c}$ are in color-singlet $D$-mesons, as in the loop shown in Fig. 2 . The effects of the medium on the self-energy of $J / \Psi$ can be incorporated using medium-modified masses for the intermediate $D$-mesons. Using a gauged $J / \Psi D \bar{D}$ effective Lagrangian, the authors of Ref. [17] found that $J / \Psi$ self-energy is positive (i.e. the mass of $J / \Psi$ is medium is larger than in free space) and therefore $J / \Psi$ would not be bound to nuclear matter.

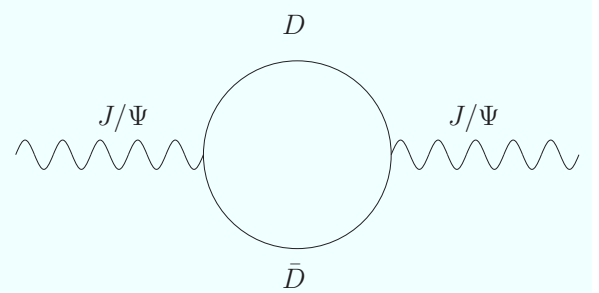

FIGURE 2. $D D$-loop contribution to the $J / \Psi$ self-energy. There are also $D D^{*}$ and $D^{*} D^{*}$ contributions.

We [4] believe that the repulsive result for the self-energy is due to the use of a gauged Lagrangian. In the present communication we show this explicitly by using a different, non-gauged Lagrangian. Specifically, we use an SU(4) extension of a $\rho \pi \pi$ Lagrangian that was used previously to calculate the $\rho$ self-energy via a $\pi \pi$ loop [18]. Our study is an ongoing project [19] where in addition to $D D$, there are also $D D^{*}$ and $D^{*} D^{*}$ intermediate states - explicit expressions and a complete discussion including the algebraically more complicated $D^{*} D^{*}$ loop contribution will be reported elsewhere [4]. 
The Lagrangian densities for $\Psi D D$ and $\Psi D D^{*}$ (we denote $J / \Psi$ by $\Psi$ ) vertices are given respectively by

$$
\mathscr{L}_{\psi D \bar{D}}=i g_{\psi D \bar{D}} \psi^{\mu}\left[\bar{D}\left(\partial_{\mu} D\right)-\left(\partial_{\mu} \bar{D}\right) D\right],
$$

and

$$
\mathscr{L}_{\psi D D^{*}}=\frac{g_{\psi D D^{*}}}{m_{\psi}} \varepsilon_{\alpha \beta \mu \nu}\left(\partial^{\alpha} \psi^{\beta}\right)\left[\left(\partial_{\mu} \bar{D}^{* v}\right) D+\bar{D}\left(\partial_{\mu} D^{* v}\right)\right] .
$$

Taking for simplicity equal masses the charged and neutral $D$ states (and the same for $D^{*}$ states) and $g_{\psi D \bar{D}}=g_{\psi D D^{*}} \equiv g$, calculation of the loop diagram in Fig. 2 leads for the $\Psi$ self-energy the expression

$$
i \Sigma^{\Psi}(k)=-\frac{8}{3} g^{2} \int \frac{d^{4} q}{(2 \pi)^{4}} \mathbf{q}^{2}\left[\Delta_{D}(q)+\Delta_{D^{*}}(q)\right] \Delta_{D}(q-k) F\left(\mathbf{q}^{2}\right),
$$

where $\Delta_{D}(q)=1 /\left(q^{2}-m_{D}^{2}+i \varepsilon\right)$, and $F\left(\mathbf{q}^{2}\right)$ is a form factor.

Medium effects on the intermediate $D$ and $D^{*}$ mesons are calculated in the context of the quark-meson-coupling (QMC) model [20]. This is a relativistic mean field model of nuclear matter, in which effective meson degrees of freedom are coupled directly to quarks confined in a bag (or confined by a potential [21]). Using scalar-isoscalar $\sigma$, vector-isoscalar $\omega$, and vector-isovector $\rho$ mean fields that couple(with adjustable coupling constants) to the light $u$ and $d$ quarks is sufficient to achieve a reasonable description of saturation properties of nuclear matter as well as energy levels of finite nuclei - a review on the model and a large collection of results can be found in Ref. [22].

The $J / \Psi$ mass is determined from the relation

$$
m_{\Psi}^{2}=\left(m_{\Psi}^{0}\right)^{2}+\Sigma\left(q^{2}=m_{\psi}^{2}\right),
$$

where $m_{\Psi}^{0}$ is the bare mass. For the form factor needed in Eq. (6) we use a dipole at each vertex parametrized by a cutoff $\Lambda$. We fix the vacuum values of the masses to $m_{\Psi}=3096.9 \mathrm{MeV}$ and $m_{D}=1867.2 \mathrm{MeV}, m_{D^{*}}=2008.6 \mathrm{MeV}$, and determine $m_{\Psi}^{0}$ for different $\Lambda$. Using $g_{J / \Psi D D}=g_{J / \Psi D D^{*}}=7.7$, we obtain that the difference between the in-medium and vacuum masses of $J / \Psi, \Delta m_{\Psi}=m_{\Psi^{*}}-m_{\Psi}$, can be parameterized in terms of the nuclear density as

$$
\Delta m_{\Psi}=-m_{\Psi}\left(1-\alpha_{\Lambda} \rho / \rho_{0}\right),
$$

where $\rho_{0}$ is the normal nuclear matter density, and $\alpha_{\Lambda}$ is a number that depends on the cutoff value. This parameterization is approximately valid up to $\rho \simeq \rho_{0}$. We believe that reasonable values for $\Lambda$ are between the range of $1000 \mathrm{MeV}$ and $2000 \mathrm{MeV}$ and have obtained, for example, $\alpha_{1000}=0.9984, \alpha_{1500}=0.9976, \alpha_{2000}=0.9968$. This translates to a $\Delta m_{\Psi} \simeq-7.5 \mathrm{MeV}$ for $\Lambda=1500 \mathrm{MeV}$.

If one considers $\Delta m_{\Psi}$ as a potential, one natural question is that whether a mass shift of $7.5 \mathrm{MeV}$ would be enough to bind $J / \Psi$ to a nucleus. From elementary quantum mechanics, one knows that for an attractive spherical well of radius $a$ and depth $V_{0}$, the condition for the existence of an $s$-wave bound state of a particle of mass $m$ is $V_{0}>\pi^{2} \hbar^{2} / 8 m a^{2}$. Using for $m=m_{\psi}$ and $a=5 \mathrm{fm}$, one obtains $V_{0}>1 \mathrm{MeV}$. 


\section{CONCLUSIONS}

Initially we have shown that properties of charmonia at finite temperature calculated within potential models depend crucially on how string breaking is implemented. In particular, we have shown that when a sudden string breaking potential is used, charmonium radii are unaffected by temperature up to the vicinity of a critical temperature $T_{c}$ when the string breaks, but as this temperature is exceeded the radii increase suddenly. This is in opposition to the most commonly used smooth screening potentials. Implications of the results for the phenomenology of charmonium interactions in medium, in particular for scattering dissociation processes, were discussed.

Next, we explored the possibility of the existence of an exotic nuclear bound state in which a charmonium state produced close to threshold is captured in matter. Attraction of $J / \Psi$ to nuclear matter is achieved via the excitation of an intermediate state of $D$ and $D^{*}$ mesons. Sufficient attraction for the formation of a $J / \Psi$-nucleus bound state is achieved when the masses of the $D$ and $D^{*}$ mesons are made density dependent according to the QMC model. Our study was based on a non-gauged Lagrangian for the $J / \Psi D \bar{D}$ vertice.

\section{ACKNOWLEDGMENTS}

The author would like to thank A.W. Thomas, K. Tsushima, A. Valcarce, and J. Vijande for the collaboration. Work partially financed by FAPESP and CNPq (Brazilian agencies).

\section{REFERENCES}

1. T. Matsui, and H. Satz, Phys. Lett. B 178, 416-422 (1986).

2. S. J. Brodsky, I. A. Schmidt, and G. F. de Teramond, Phys. Rev. Lett. 64, 1011 (1990).

3. J. Vijande, G. Krein, and A. Valcarce, Eur. Phys. J. A 40, 89 (2009).

4. G. Krein, A.W. Thomas, and K. Tsushima, in preparation.

5. G. S. Bali, H. Neff, T. Duessel, T. Lippert, and K. Schilling, Phys. Rev. D 71, 114513 (2005).

6. M. Asakawa, and T. Hatsuda, Phys. Rev. Lett. 92, 012001 (2004).

7. S. Datta, F. Karsch, P. Petreczky, and I. Wetzorke, Phys. Rev. D 69, 094507 (2004).

8. T. Umeda, K. Nomura, and H. Matsufuru, Eur. Phys. J. C 39S1, 9 (2005).

9. A. Mocsy, Nucl. Phys. A 830, 411c (2009).

10. C. A. Dominguez, M. Loewe, and J. C. Rojas, Braz. J. Phys. 38 (2008) 437.

11. C. A. Dominguez, M. Loewe, and J. C. Rojas, JHEP 0708, 040 (2007).

12. D. Hadjimichef, G. Krein, S. Szpigel, and J. S. da Veiga, Annals Phys. 268, 105 (1998).

13. C. Y. Wong, E. S. Swanson, and T. Barnes, Phys. Rev. C 62, 045201 (2000).

14. J. P. Hilbert, N. Black, T. Barnes, and E. S. Swanson, Phys. Rev. C 75, 064907 (2007).

15. J. Haidenbauer, G. Krein, U. G. Meissner, and A. Sibirtsev, Eur. Phys. J. A 33, 107 (2007).

16. M.E. Peskin, Nucl. Phys. B 156365 (1979).

17. S.H. Lee, and C.M. Ko, Phys. Rev. C 67, 038202 (2003).

18. D. B. Leinweber, A. W. Thomas, K. Tsushima and S. V. Wright, Phys. Rev. D 64, 094502 (2001).

19. K. Tsushima, AIP Conf. Proc. 1182, 886 (2009).

20. P. A. M. Guichon, Phys. Lett. B 200, 235 (1988).

21. M.E. Bracco, G. Krein, and M. Nielsen, Phys. Lett. B 432, 258 (1998).

22. K. Saito, K. Tsushima, and A. W. Thomas, Prog. Part. Nucl. Phys. 58, 1 (2007). 
Copyright of AIP Conference Proceedings is the property of American Institute of Physics and its content may not be copied or emailed to multiple sites or posted to a listserv without the copyright holder's express written permission. However, users may print, download, or email articles for individual use. 